\title{
Shielding Effectiveness Data on Commercial Thermoplastic Materials
}

\author{
Urban Lundgren, Member, IEEE, Jonas Ekman, Member, IEEE, and Jerker Delsing, Member, IEEE
}

\begin{abstract}
Ten different commercially available conductive thermoplastic materials have been tested for near- and far-field shielding effectiveness (SE). Far-field SE was tested using a modified standard measurement technique to provide results comparable with the company-provided data. Further, housings of different thermoplastic materials were constructed and equipped with an electromagnetic interference (EMI) source to model a realistic near-field $\mathrm{SE}$ situation. The SE data up to $1 \mathrm{GHz}$ is presented. Conductive thermoplastic materials with fillings of stainless steel fibers and nickel-coated carbon fibers were the two materials that offer the best far-field shielding performance. For the near-field shielding, two materials with filling of stainless steel fibers were the best performing ones. A thermoplastic with polycarbonate (PC) base and stainless steel content of 1.5 vol\% showed the best combined farand near-field shielding results.
\end{abstract}

Index Terms-Conductive composite material, far field, IEEESTD 299, near field, shielding effectiveness (SE), thermoplastic.

\section{INTRODUCTION}

$\mathbf{F}$ OR encapsulating electronic devices, boxes of metal sheets have been used earlier. During the last two decades, plastic materials have replaced metal in applications where decreased weight, flexibility, and aesthetic appeal are important design issues. In the last couple of years, internal clock frequencies in electronic circuits have increased to several $\mathrm{GHz}$, making electromagnetic compatibility (EMC) and thermal management increasingly important issues. Thus, controlling the emitted radio frequency electromagnetic emissions is important. Also, circuits must be protected from the electromagnetic energy in its environment. The circuits are protected by including filters in the circuit design and using encapsulation with electromagnetic shielding capabilities.

There are a number of methods available to make encapsulations of plastic materials offering some degree of electromagnetic shielding [1]. One such method includes surface treatment of nonconductive plastic such as conductive paint, vacuum metallization, etc. Another method is to mix conductive particles with plastic before the encapsulation product is formed. A great advantage with this method is that the number of production steps are reduced.

One problem that arises for the EMC engineer is the selection of an encapsulation technique that offers the desired degree of electromagnetic shielding for a new electronic device.
Manufacturers of different conductive (thermoplastic) materials sometimes specify the shielding performance of their materials in an application according to standardized measurement methods, but deviations from the exact standard often occur. This deviation makes the comparisons between different manufacturers hard. The standardized method just gives a hint of what the shielding performance can be for the same material in an electronic device encapsulation application.

Another solution is the theoretical calculation or numerical modeling of electromagnetic shielding effectiveness (SE). This requires the knowledge of electrical and electromagnetic material parameters that also have to be provided or measured. However, while the calculations are restricted to simple geometries [2], the possibilities with numerical modeling can be expected to increase with future software such as those discussed in [3].

To measure the plane-wave SE for a small sample of a material, several methods have been published; for a review, see [4] and [5]. The SE evaluation of flat, thin samples using the ASTM D4935 [6] is cost effective especially for the manufacturers of conductive thermoplastics. The test involves a circular split coaxial transmission line (flanged coaxial holder) and is often referred to by conductive composite material manufacturers. A problem with this kind of measurement method is that it seldom reflects the real situation. When a material is used in an enclosure for an electronic device, it is usually exposed to different electromagnetic field conditions than that in the standardized test method.

The SE data of commercially available conductive thermoplastic materials are seldom published other than in product specifications from the manufacturers. Also, different manufacturers use different measurement methods, making the comparisons hard. Thus, we decided to evaluate the electromagnetic SE for commercially available thermoplastic materials. Ten materials were chosen and samples were manufactured for analysis using two measurement methods, similar to [7]. Section II presents the composition of the chosen materials, while the measurement techniques are presented and discussed in Section III. Section IV presents the recorded results. Also, for some cases, manufacturers' data were available for comparison. The conclusions from the comparisons are drawn in Section V.

\section{PRESEntation OF Thermoplastic MATERIALS}

This paper presents measurements on ten thermoplastic materials. The raw materials were bought from three large manufacturers and prepared according to manufacturer instructions.
Manuscript received September 23, 2005; revised April 28, 2006

J. Ekman and J. Delsing are with EISLAB, Luleå University of Technology, Luleå S-971 87, Sweden.

Digital Object Identifier 10.1109/TEMC.2006.879352 


\section{A. Manufacturer 1}

Manufacturer 1 offers compounds that combine thermoplastics [acrylonitrile-butadiene-styrene (ABS), polycarbonate (PC), PC/ABS, and polypropylene (PP)] with stainless steel fibers in relatively low concentrations. The materials are typically used for electromagnetic interference (EMI) protection (housing) of electronic components/systems including LAN connection boxes and credit card payment devices. The following materials were used:

- Material 1:1. ABS with stainless steel content of $1.5 \mathrm{vol} \%$ corresponding to $10 \mathrm{wt} \%$, contains flame retardants;

- Material 1:2. PP with stainless steel content of 1.5 vol.\% corresponding to $12 \mathrm{wt} \%$;

- Material 1:3. PC with stainless steel content of 1.5 vol.\% corresponding to $9 \mathrm{wt} \%$, contains flame retardants;

- Material 1:4. PC/ABS with stainless steel content of $1 \mathrm{vol} \%$ corresponding to $7 \mathrm{wt} \%$, contains flame retardants;

- Material 1:5. ABS with stainless steel content of 1 vol. $\%$ corresponding to $7 \mathrm{wt} \%$, contains flame retardants.

\section{B. Manufacturer 2}

Manufacturer 2 offers different kinds of plastic products to match specific applications demanding EMI shielding, hightemperature protection, and wear durability. Typically, carbon fibers, stainless steel fibers, or nickel-coated carbon fibers are used in a thermoplastic matrix to provide the necessary shielding. The following materials were used:

- Material 2:1. PC/ABS with nickel-coated carbon fibers content of $20 \mathrm{wt} \%$, contains flame retardants;

- Material 2:2. PP with stainless steel fibers content of $15 \mathrm{wt} \%$;

- Material 2:3. PC with stainless steel fibers content of $15 \mathrm{wt} \%$, contains flame retardants;

- Material 2:4. Nylon-6,6 polyhexamethylene-adipamide (PA) with nickel-coated carbon fibers content of $20 \mathrm{wt} \%$.

\section{Manufacturer 3}

Manufacturer 3 offers stainless steel fibers that are used as fillers for electrically conductive plastics. The chopped fibers are bound together with polymeric binders specific for various polymer resins. The following materials were used:

- Material 3:1. Stainless steel fibers mixed in ABS with a fiber content of $15 \mathrm{wt} \%$.

\section{SE MEASUREMENT TECHNIQUES}

For the measurement of electromagnetic SE, various techniques offer different dynamic ranges in different frequency intervals. A number of standardized methods exist; however, in practical use, tailor made test fixtures often deviate from the design standards, making the measured data specific to the modified fixture and comparisons less significant. Also, the demands on the preparation of test samples may influence the choice of the measurement method.

We have chosen to use two measurements methods: First, given in Section III-A, is a far-field shielding measurement that

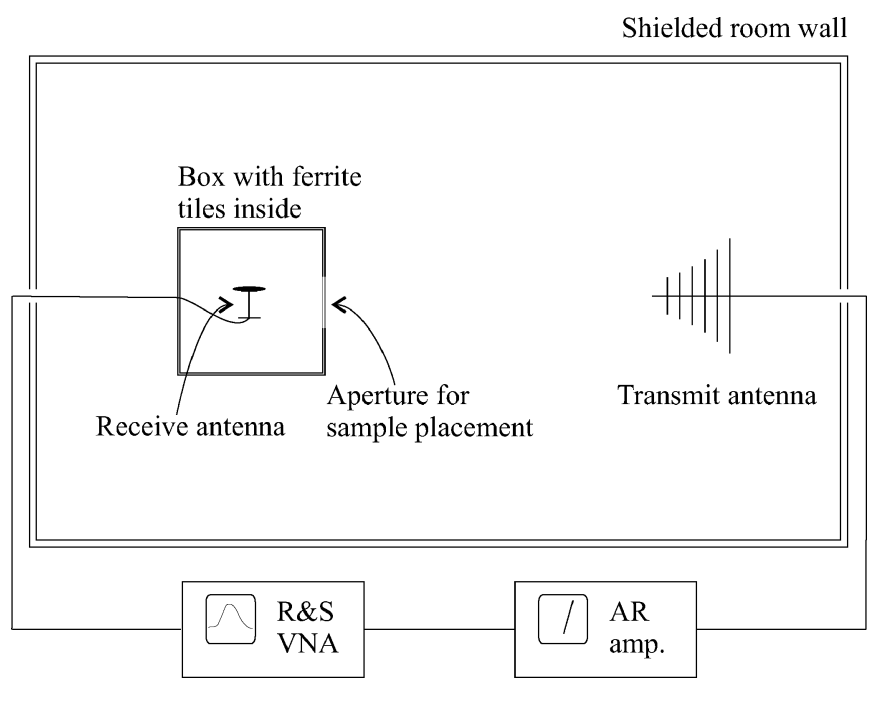

Fig. 1. Schematic description over modified MIL-STD-285 method utilized for far-field SE measurements.

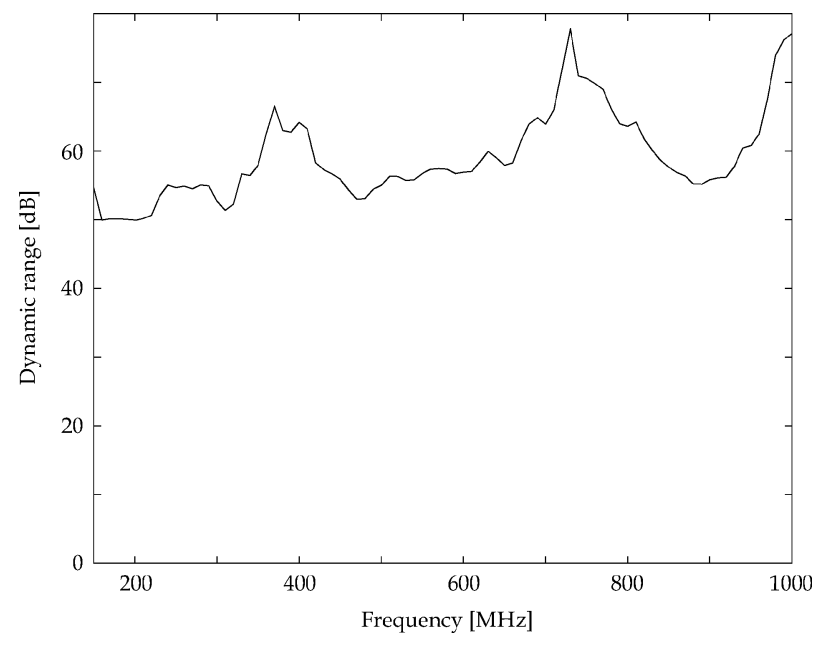

Fig. 2. Dynamic range for far-field SE measurement test setup.

is based on the standardized methods in [10]. Second, given in Section III-B, is a generic approach to totally encapsulate a transmitter in its near field with the material under test. The intention in the second method is to simulate an application of the material where it is used for the encapsulation of a working electronic device. A comparison has been made between the far-field SE measurement method and the application imitating the near-field method [8].

\section{A. Far-Field SE Measurements, Modified MIL-STD 285 Type Measurement}

Earlier, the foundation of SE measurements was the American military standard MIL-STD-285 [9] from 1956. This method in now withdrawn and replaced with IEEE-STD 299 [10]. The test method requires a shielded enclosure with an open window where test objects can be placed. The transmitter and receiver antennas are located on separate sides of the window and directed towards each other at a fixed distance. The transmitter transmits at a constant power, and the receiver measures the 


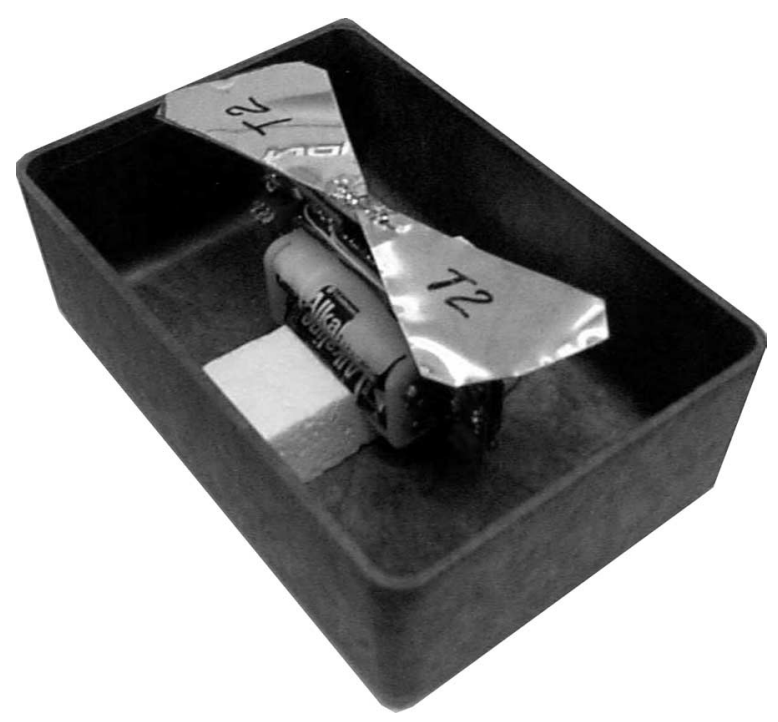

Fig. 3. Transmitter placed in box made of a thermoplastic material.

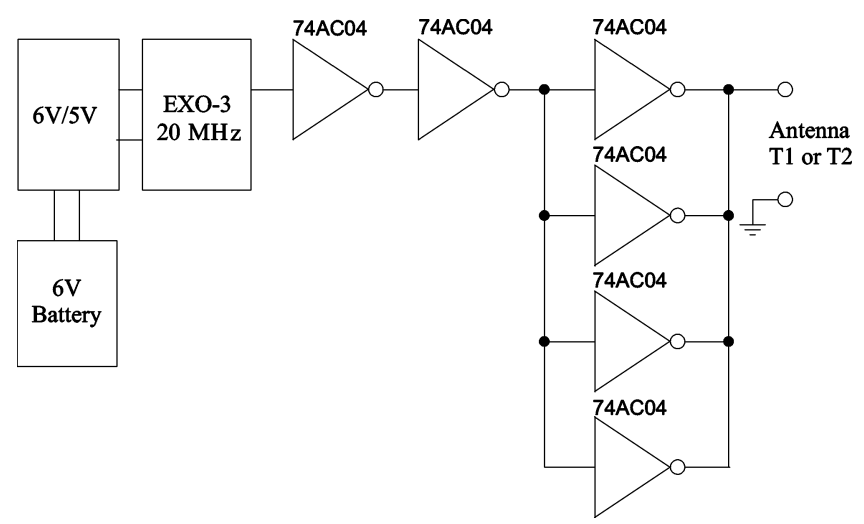

Fig. 4. Schematic for battery-powered transmitter placed in boxes.

transferred power with and without the test object mounted in the aperture. According to [10] (and the upcoming 2005 release), the SE is calculated from the magnetic field, the electric field, and the detected power according to the following:

- Low-range, $9 \mathrm{kHz}$ to $20 \mathrm{MHz}$ (extendable up to $50 \mathrm{~Hz}$ ), utilizing loop antennas. The form for expressing the SE is in terms of magnetic-field performance

$$
\mathrm{SE}_{H}=20 \log _{10} \frac{\left|H_{1}\right|}{\left|H_{2}\right|}
$$

where $H_{1}$ is the magnetic field in the absence of the material, and $\mathrm{H}_{2}$ is the magnetic field with the material.

- Mid-range, 20 to $300 \mathrm{MHz}$, utilizing dipole or horn antennas. The form for expressing the SE is in terms of electricfield performance

$$
\mathrm{SE}_{E}=20 \log _{10} \frac{\left|E_{1}\right|}{\left|E_{2}\right|}
$$

where $E_{1}$ is the electric field in the absence of the material, and $E_{2}$ is the electric field with the material.

- High-range, $300 \mathrm{MHz}$ to $1.7 \mathrm{GHz}$ and 1.7 to $1.8 \mathrm{GHz}$ (extendable up to $100 \mathrm{GHz}$ ), utilizing horn antennas. The

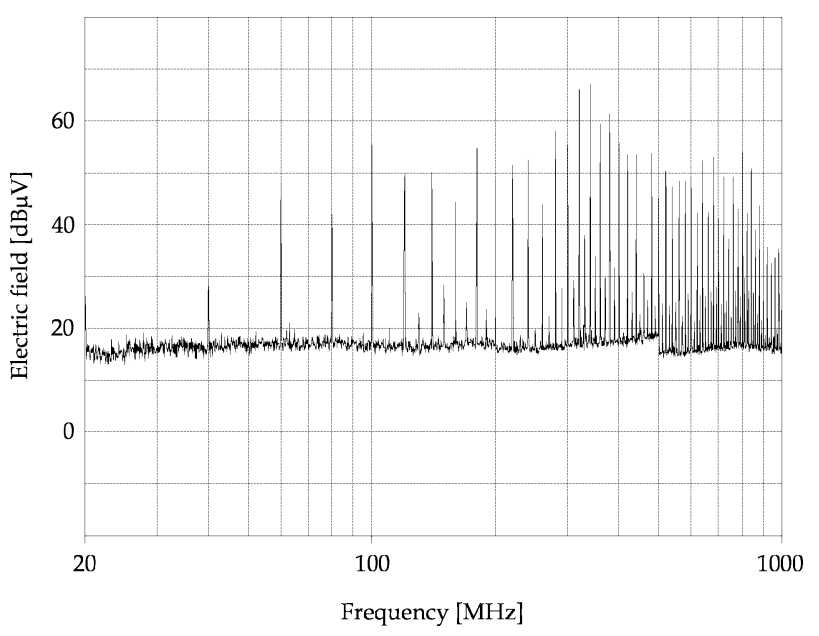

Fig. 5. Radiated electric field from battery-powered transmitter.

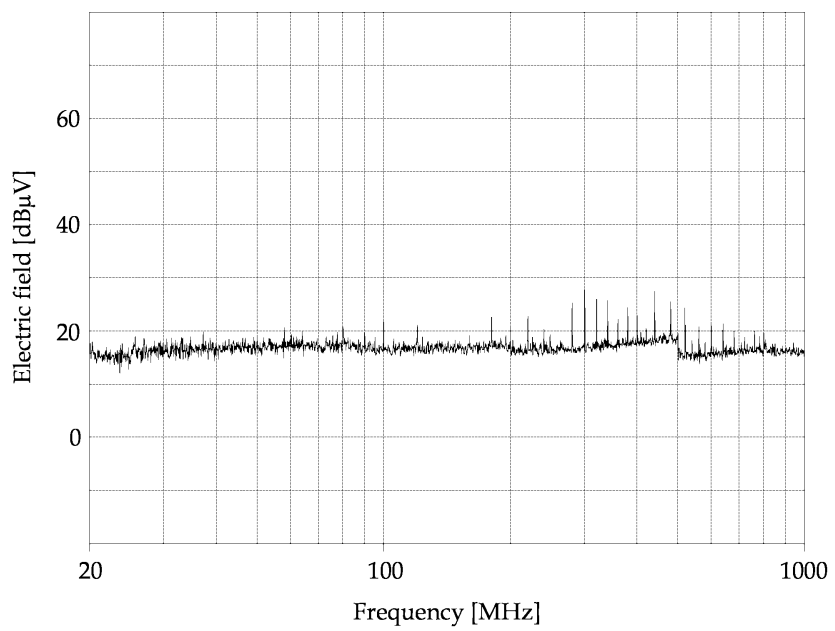

Fig. 6. Example of radiated electric field from battery-powered transmitter when placed inside one box.

form for expressing the $\mathrm{SE}$ is in terms appropriate to the detector indication by either (2) or by one of the following:

$$
\mathrm{SE}_{P}=10 \log _{10} \frac{P_{1}}{P_{2}}
$$

where $P_{1}$ is the power detected in the absence of the material, and $P_{2}$ is the power detected with the material present or

$$
\mathrm{SE}_{P}=A_{1}-A_{2}
$$

where $A_{1}$ is the source attenuator setting, in decibels, for a measurable output of a specified detector in the absence of the material, and $A_{2}$ is the source attenuator setting for the same output of the detector with the material present.

Additional considerations when measuring electromagnetic SE include

1) the possibility of cavity resonances causing considerable variability of data;

2) nonlinear effects in the presence of strong emissions;

3) the dependence on antenna placement and orientation. 


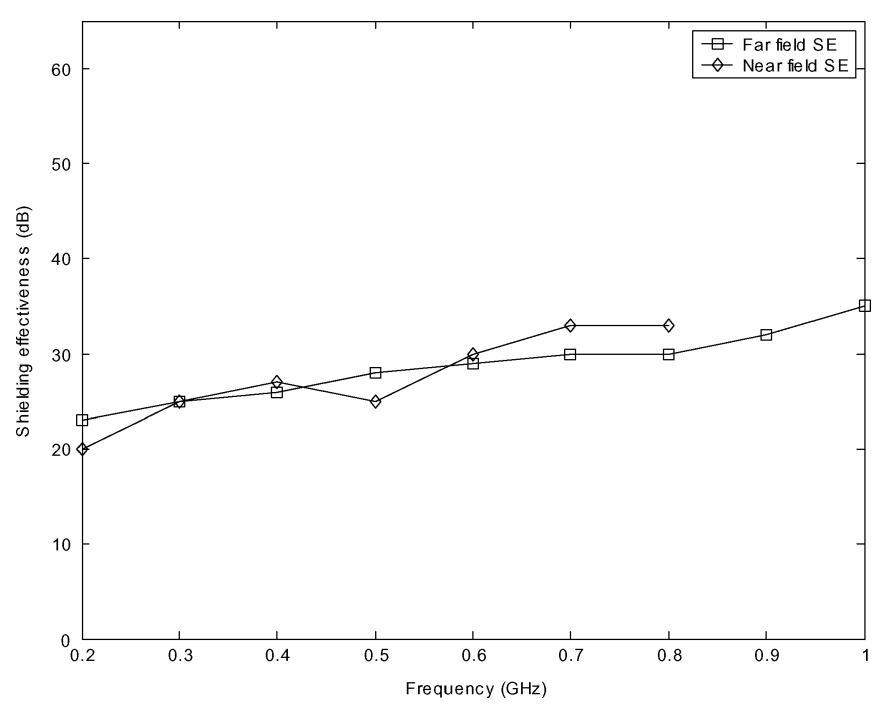

Fig. 7. Measured SE for material 1:1. The SE data for plane-wave shielding is denoted by squares. The results for the application-specific near-field SE of an electric field source are denoted by diamonds.

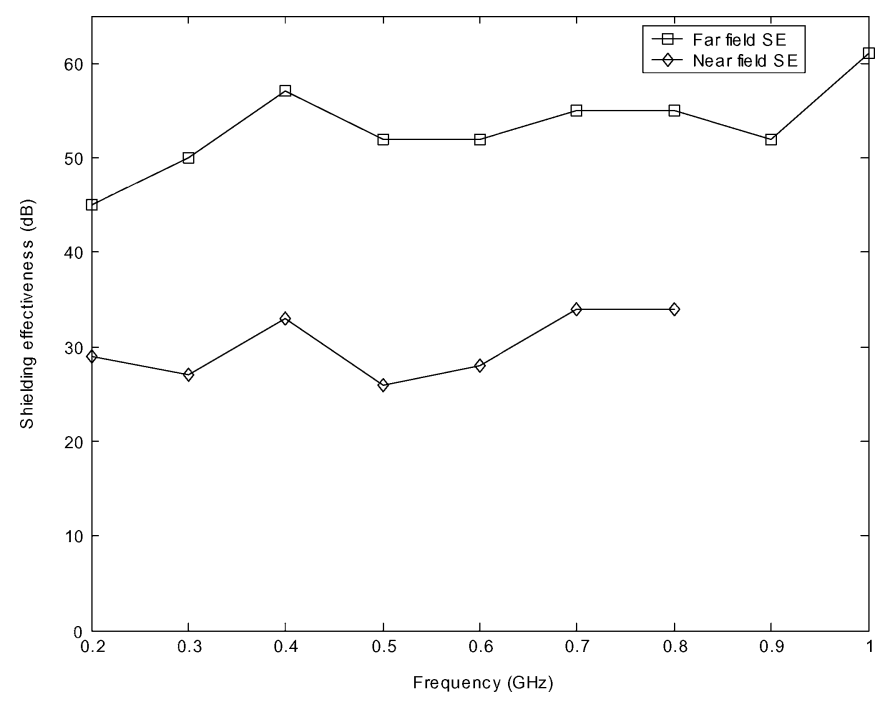

Fig. 8. Measured SE for material 1:2. The SE data for plane-wave shielding is denoted by squares. The results for the application-specific near-field SE of an electric field source are denoted by diamonds.

Improved versions of the method in MIL-STD-285 have been developed in which the problems with reflections have been minimized. A proposed method [12] involves a box, with absorbers on the inside walls, containing the transmitting antenna. There is an aperture in one wall of the box where test objects can be mounted. The box is placed in an anechoic shielded room, together with the receiving antenna. The insertion loss is determined in the same manner as mentioned earlier. This improved method has been used in this paper for the far-field SE measurements with the modifications that the receiving antenna is placed inside the box and the transmitting antenna in the room outside the box; see Fig. 1. Also, the brass box aperture and the sample holder have been adapted to the material samples available to us. Inside an anechoic shielded room, the box containing the receiver antenna is placed on a wooden table. The box is damped on the inside with ferrite tiles and has a $90 \times 90 \mathrm{~mm}$ aperture on

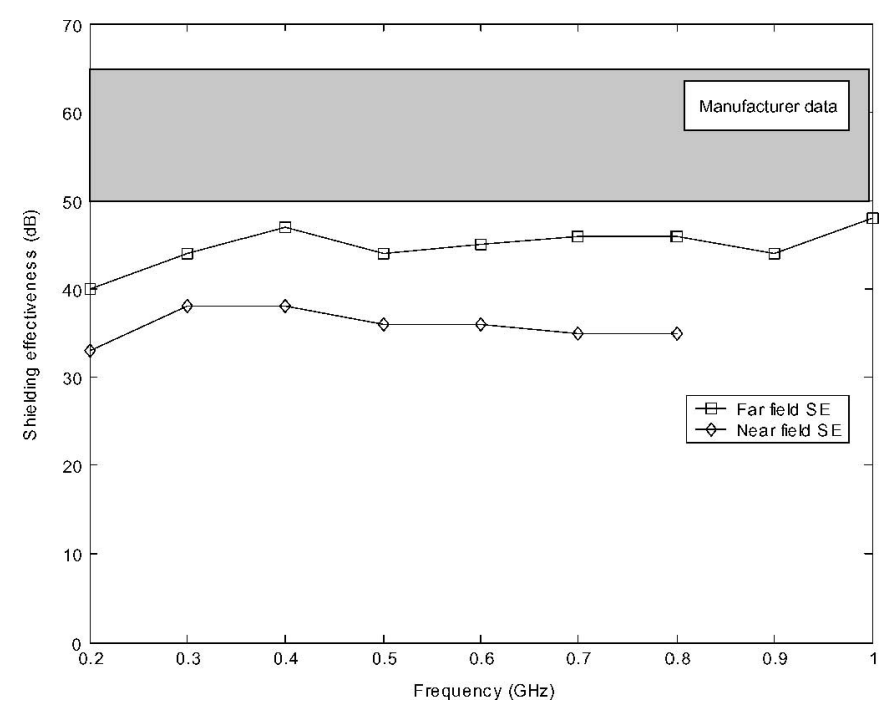

Fig. 9. Measured SE for material 1:3. The SE data for plane-wave shielding is denoted by squares. The results for the application-specific near-field SE of an electric field source are denoted by diamonds.

one side. The aperture size is determined by the thermoelectric material sample size which is $95 \times 95 \mathrm{~mm}$. A broadband transmitting antenna (Chase BiLog CBL6112) was placed 3m from the aperture, and the receiver antenna (top-loaded broadband monopole antenna) measured the transmitted power inside the box with and without a thermoplastic material in the aperture. The system, including amplifiers, is controlled by a Rohde and Schwarz ZVR network analyzer. The SE for that specific thermoplastic material is then calculated as the difference between these two measurements, as detailed in (2).

The frequency range for this test setup is $150-1000 \mathrm{MHz}$. The lower frequency limit is set to ensure far-field conditions at the front of the metallic box and by the aperture size. This aperture acts like a wave guide and attenuates the electromagnetic field under a specific cut-off frequency, resulting in measurement problems and a reduced dynamic range for lower frequencies. The upper frequency limit is set to $1000 \mathrm{MHz}$ by the upper frequency limit for the transmitter power amplifier. The dynamic range for the test setup is shown in Fig. 2 and over $50 \mathrm{~dB}$ for all investigated frequencies.

The main drawback for this setup is the small aperture size, which is only $90 \times 90 \mathrm{~mm}$. The dynamic range could be increased and the lower frequency limit could be decreased if a bigger aperture is used. However, this would require larger material samples, which are more complicated to manufacture. Other suggestions for improving the technique can be summarized as follows:

- very good cables connecting the measurement receiver and the receiver antenna, offering a higher level of shielding against the radiated incident field;

- flexible sample holder design to simplify mounting of the test sample for repeated measurements.

Especially, a flexible sample holder could improve the obtained results by enabling the measurement of averages of multiple measurements as detailed in [10]. 


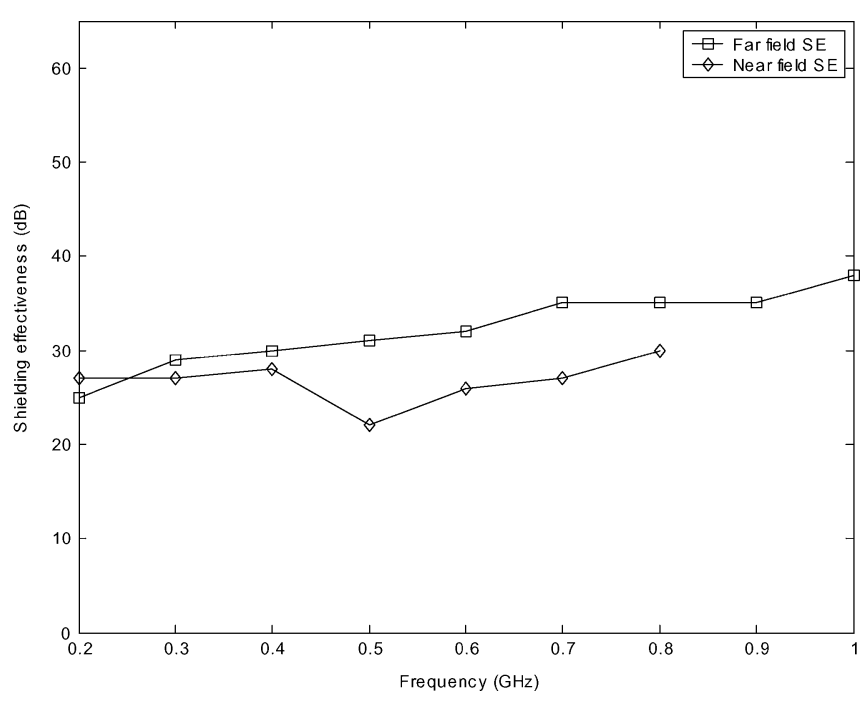

Fig. 10. Measured SE for material 1:4. The SE data for plane-wave shielding is denoted by squares. The results for the application-specific near-field SE of an electric field source are denoted by diamonds.

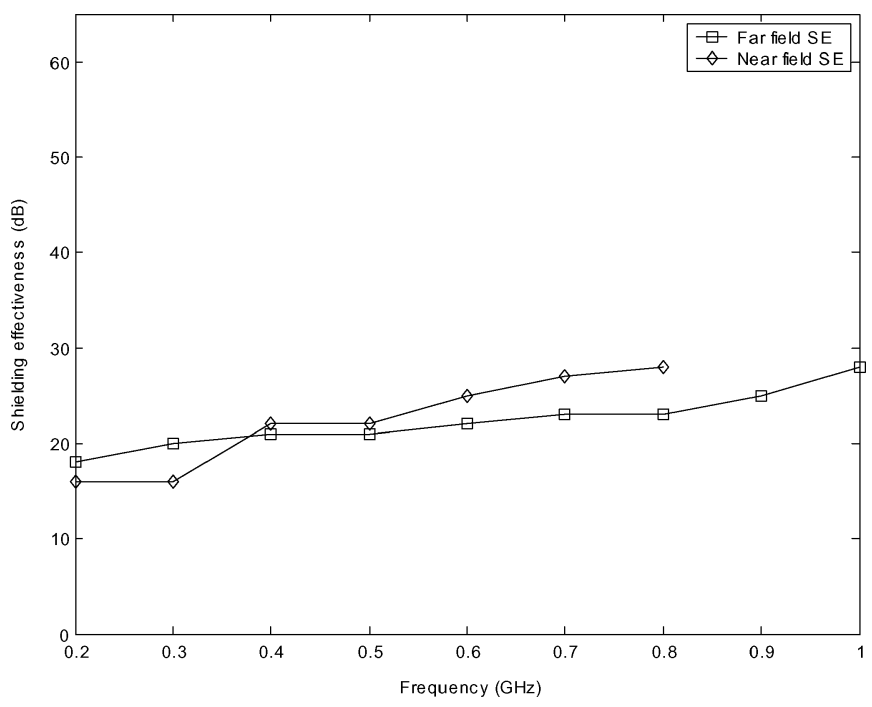

Fig. 11. Measured SE for material 1:5. The SE data for plane-wave shielding is denoted by squares. The results for the application-specific near-field SE of an electric field source are denoted by diamonds.

\section{B. Near-Field SE Measurements, Application-Specific Measurement}

The second method used a more "realistic" test setup to characterize the SE for different thermoplastic materials. A batterypowered transmitter is enclosed in a $18 \times 11 \times 12 \mathrm{~cm}$ box (see Fig. 3) made out of different materials. The transmitter, with interchangeable antennas, is constructed from a series of 74AC04 inverters and an EXO-3 crystal oscillator as shown in Fig. 4. In a reference measurement, the transmitter was placed on a table in an anechoic room and the emitted free-space signal spectra were measured at a distance of $3 \mathrm{~m}$ (Fig. 5). Then, the transmitter was enclosed in a box made of different materials, and the emitted signal spectra were measured; see a sample measurement in Fig. 6. The application-specific near-field SE was

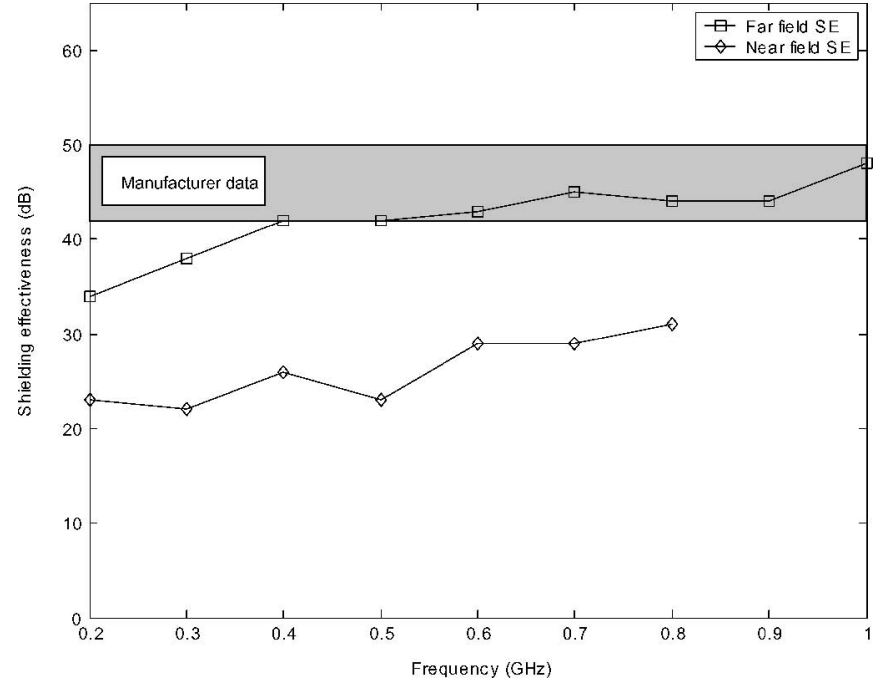

Fig. 12. Measured SE for material 2:1. The SE data for plane-wave shielding are denoted by squares. The results for the application-specific near-field SE of an electric field source are denoted by diamonds.

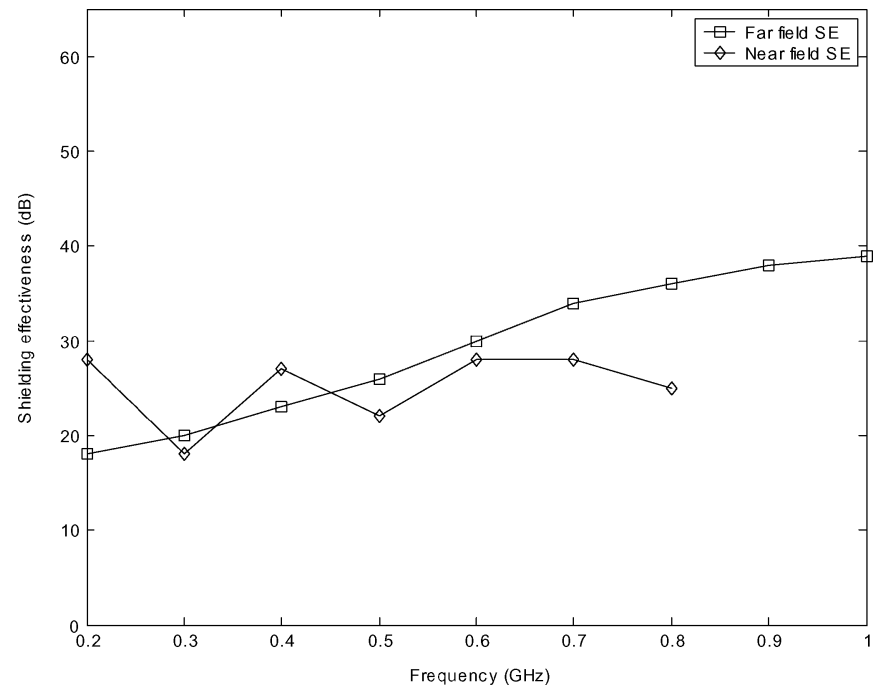

Fig. 13. Measured SE for material 2:2. The SE data for plane wave shielding are denoted by squares. The results for the application-specific near-field SE of an electric field source are denoted by diamonds.

then calculated as the difference between the measured signal spectra.

Regarding the near-field SE, both high- and low-impedance fields can be considered. However, the materials covered in this study are not expected to offer high SE for $H$ field. Rather, the near-field shielding of the electric field is of interest and needs to be addressed. Further, in the near field, the high-impedance source is loaded by the conductive shielding material (the box) so that the radiator input impedance is altered. In the method used, this alteration is considered to be a fact that resembles a real application situation and cannot be avoided. This means that the results from these measurements are "unique" to this test setup and cannot be directly applied to other shielding applications; thus, we use the term application-specific near-field SE. 


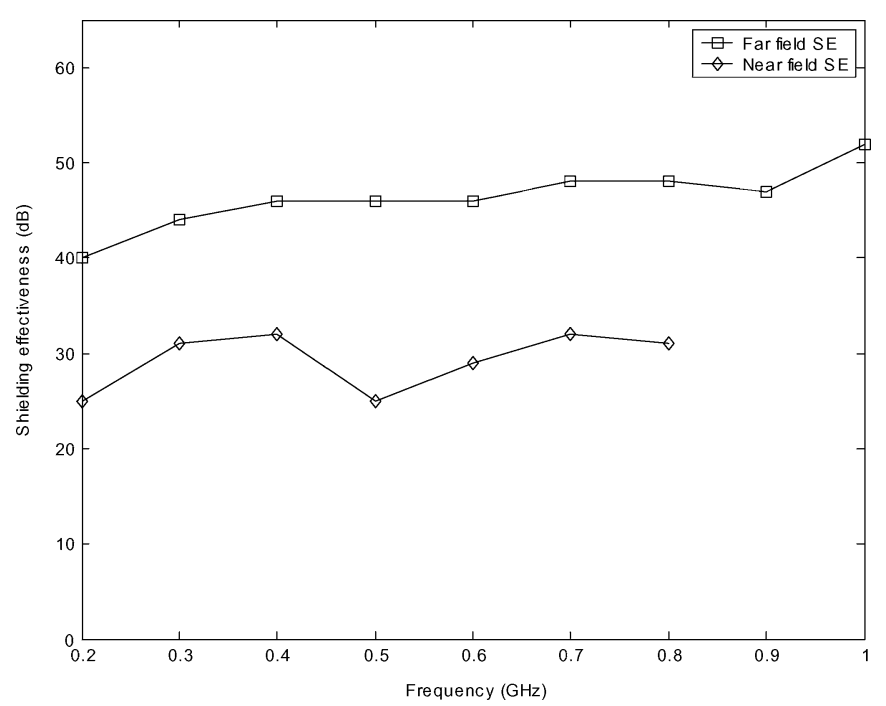

Fig. 14. Measured SE for material 2:3. The SE data for plane-wave shielding are denoted by squares. The results for the application-specific near-field SE of an electric field source are denoted by diamonds.

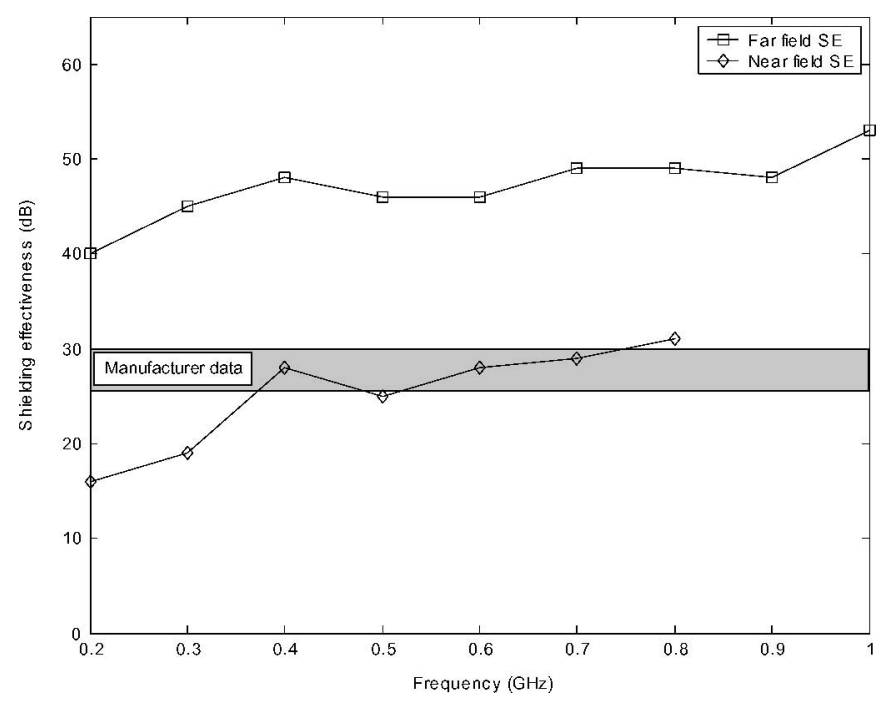

Fig. 15. Measured SE for material 2:4. The SE data for plane-wave shielding are denoted by squares. The results for the application-specific near-field SE of an electric field source are denoted by diamonds.

The low level of output power from the battery-powered transmitter gives an undesired consequence of small dynamic range for this method. The measured data from this method presented in this paper cover the frequency interval 200-800 MHz. Above $800 \mathrm{MHz}$, the output power was not sufficient.

\section{RESULTS}

\section{A. Measurement Results}

The results from the two different measurements are shown in parallel in Figs. 7-16 and collected for a set of frequencies in Table I.

The measurements clearly show SE, from $17 \mathrm{~dB}$ to more than $50 \mathrm{~dB}$, for different materials. Four of the ten materials offer a far-field SE greater than $40 \mathrm{~dB}$ in the entire frequency range.

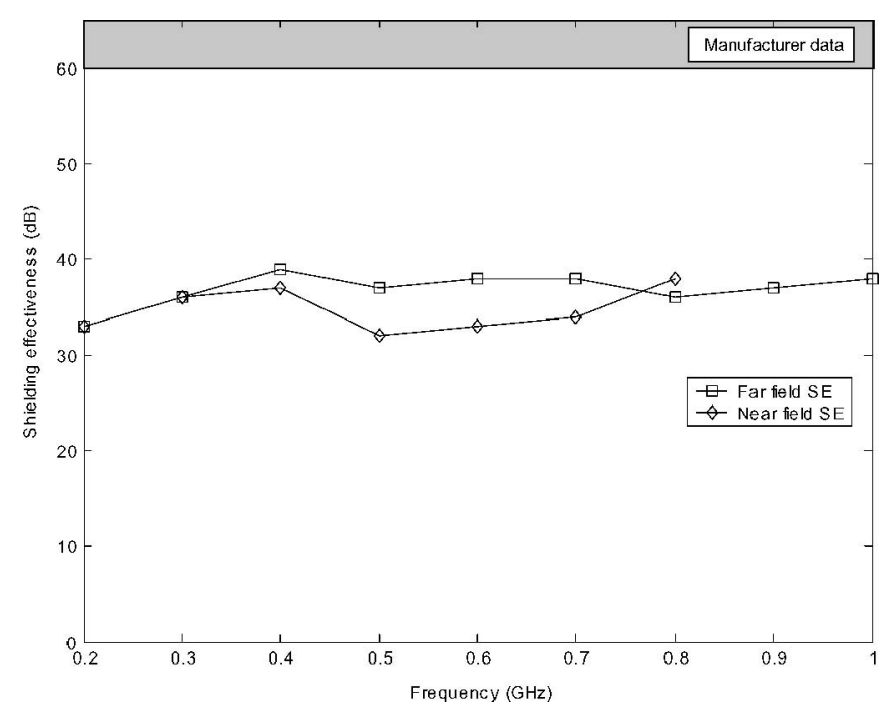

Fig. 16. Measured SE for material 3:1. The SE data for plane-wave shielding are denoted by squares. The results for the application-specific near-field SE of an electric field source are denoted by diamonds.

Among these materials, the application-specific near-field SE differs significantly. All curves show an increased SE for higher frequencies, and the reason can be the test setup and the small aperture size. As shown in Fig. 2, the dynamic range for the setup also increases with the frequency. Another possible explanation for the higher performance of the samples is the nature of the material. Testing performed on nonwoven materials and vapor-deposited materials (such as on glass) tend to show higher performance at higher frequency.

The measurement accuracy for the far-field SE measurements is hard to estimate because only two measurements were made for each material sample. Up to $\pm 6 \mathrm{~dB}$ deviation was observed when measurements were repeated. This random error may, to some extent, be explained by nonrepeatable effects from the mounting of the test samples over the aperture. Other problems with this type of measurement method are also known [11]. A bias error of $\pm 2 \mathrm{~dB}$ is expected in this measurement. The measurements were not repeated sufficient number of times to do a proper statistical analysis of the distribution of the measurement results because the sample holder was difficult to work with.

\section{B. Comparison With Manufacturer Data}

For comparison, manufacturer data for some thermoplastics were collected from web pages and product specifications.

For material 1:3 (3-mm-thick sample), the manufacturer reported the far-field SE to be $50-65 \mathrm{~dB}$ using the ASTM D4935 test method. The frequency range is unspecified, but $30 \mathrm{MHz}$ to $1.8 \mathrm{GHz}$ is a typical range for this method. The measured far-field SE data presented in this paper lies in the range 40-48 $\mathrm{dB}$ for a 3-mm-thick sample in the frequency range $200 \mathrm{MHz}$ to $1 \mathrm{GHz}$.

For material 2:1 (0.120 in thick corresponding to $3.05 \mathrm{~mm})$, the manufacturer reported the far-field SE to be $42-50 \mathrm{~dB}$ using the ASTM D4935 test method. The frequency range is $100 \mathrm{MHz}$ to $1.5 \mathrm{GHz}$. The measured far-field SE data presented in this 
TABLE I

APPLICATION-SPECIFIC NEAR-FIELD SE AND FAR-FIELD SE FOR THE THERMOPLASTIC MATERIALS FOR A SET OF FREQUENCIES

\begin{tabular}{|c|c|c|c|c|c|c|c|c|}
\hline \multirow[t]{2}{*}{ Material } & & & \multicolumn{3}{|c|}{ Far field SE } & \multicolumn{3}{|c|}{ Near field SE } \\
\hline & & & $300 \mathrm{MHz}$ & $600 \mathrm{MHz}$ & $800 \mathrm{MHz}$ & $300 \mathrm{MHz}$ & $600 \mathrm{MHz}$ & $800 \mathrm{MHz}$ \\
\hline 1.1: ABS & $+\mathrm{SS}^{\diamond}$ & $10 \%$-wt & 25 & 29 & 30 & 25 & 30 & 33 \\
\hline 1.2: PP & $+\mathrm{SS}$ & $12 \%$-wt & 50 & 52 & 55 & 27 & 28 & 34 \\
\hline 1.3: PC & $+\mathrm{SS}$ & $9 \%$-wt & 44 & 45 & 46 & 38 & 36 & 35 \\
\hline 1.4: PC/ABS & $+\mathrm{SS}$ & $7 \%$-wt & 29 & 33 & 35 & 27 & 26 & 30 \\
\hline 1.5: ABS & $+\mathrm{SS}$ & $7 \%$-wt & 20 & 22 & 23 & 17 & 25 & 28 \\
\hline 2.1: $\mathrm{PC} / \mathrm{ABS}$ & $+\mathrm{Ni}-\mathrm{C}^{*}$ & $20 \%$-wt & 37 & 43 & 44 & 22 & 29 & 31 \\
\hline 2.2: PP & $+\mathrm{SS}$ & $15 \%-\mathrm{wt}$ & 20 & 30 & 36 & 18 & 27 & 25 \\
\hline 2.3: PC & $+\mathrm{SS}$ & $15 \%$-wt & 44 & 46 & 48 & 32 & 29 & 31 \\
\hline 2.4: PA & $+\mathrm{Ni}-\mathrm{C}$ & $20 \%$-wt & 45 & 46 & 49 & 19 & 28 & 31 \\
\hline 3.1: ABS & $+\mathrm{SS}$ & $15 \%$-wt & 36 & 38 & 36 & 36 & 33 & 38 \\
\hline
\end{tabular}

$\diamond$ - Stainless steel (SS) fibres.

* - Nickel coated carbon (Ni-C) fibres.

paper lies in the range 35-49 $\mathrm{dB}$ for a 3-mm sample in the frequency range $200 \mathrm{MHz}$ to $1 \mathrm{GHz}$.

For material 2:4 (0.110 in thick corresponding to $2.79 \mathrm{~mm})$, the manufacturer reported the far-field SE to be $26-30 \mathrm{~dB}$ using the ASTM D4935 test method. The frequency range is $100 \mathrm{MHz}$ to $1.5 \mathrm{GHz}$. The measured far-field SE data presented in this paper lies in the range $40-53 \mathrm{~dB}$ for a 3-mm sample in the frequency range $200 \mathrm{MHz}$ to $1 \mathrm{GHz}$.

Material 3:1 is stated by the manufacturer to offer more than $60 \mathrm{~dB}$ electromagnetic SE in the frequency range $30 \mathrm{MHz}$ to $1 \mathrm{GHz}$. This value corresponds to that of a conductive composite with $15 \%$ stainless steel fiber content. The test method and sample thickness are not specified. The measured far-field SE data presented in this paper lies in the range 33-38 dB for a 3 -mm sample in the frequency range $200 \mathrm{MHz}$ to $1 \mathrm{GHz}$.

\section{CONCLUSION}

When comparing the near- and far-field SEs for the thermoplastic materials, the following are noted.

- Material 1:2 offers the best far-field SE.

- Material 1:3 and material 3:1 offer the best applicationspecific near-field SE, forcing the signal from the EMI source (transmitter) below the noise floor.

- Material 1:5 is the material with the lowest level of SE for both near and far fields.

- Material 1:3 is the best material for the combined SE.

Considering that the application-specific near-field SE measurement imitates the use of material in an application, it is disappointing to see how poor an indicator the far-field SE results are when a material selection for an enclosure must be made. In cases where manufacturer data were available, agreement was quite good with the near-field method in one instance, while the difference was close to $30 \mathrm{~dB}$ in the other three instances. However, we stress that the application-specific near-field SE results are strongly correlated to the characteristics of the source (Figs. 3 and 4), the placement of the source inside the box, and the geometry of the box/barrier.
Polymers with a $20 \%$ fill of nickel-coated carbon fibers perform similar to the polymers with $15 \%$ fill of stainless steel in far-field measurement. Choice of the base polymer seems to influence the performance of the conductive plastic material. Particularly, the ABS base polymer gave lower far-field $\mathrm{SE}$ values that other base polymers with the same amount of conductive filler.

In this study, only material 3:1 was mixed when the plastic samples were produced, while all others were premixed compounded grains. This may have caused poor fiber distribution in the mold and gives some explanation why poor far-field results were observed for the third manufacturer material. Material 2:2 showed lower SE performance than expected when compared to other materials with the same base polymer and the amount of conductive filler. The reason for the poor performance is unclear and has not been studied.

Materials containing stainless steel fibers show better application-specific near-field SE than materials with nickelcoated carbon fibers, even though the stainless steel fiber's filler percentage is lower. The material 2:2 shows unexpected poor application-specific near-field SE compared to the stainless steel filler percentage. The reason for the poor SE has not been studied.

Material 3:1 containing stainless steel fibers performed relatively well for near-field SE than for far-field SE. The reason for this has not been studied but may be caused by nonuniform fiber distribution in the mold.

\section{REFERENCES}

[1] B. D. Mottahed and S. Manoochehri, "A review of materials, modeling and simulation, design factors, testing, and measurements related to electromagnetic interference shielding," Polymer-Plastics Technol. Eng., vol. 34, no. 2, pp. 271-346, Mar. 1995.

[2] P. R. Bannister, "New theoretical expressions for predicting shielding effectiveness for the plane shield case," IEEE Trans. Electromagn. Compat., vol. EMC-10, pp. 2-7, Mar. 1968.

[3] CST Microwave Studio. (2005). Computer Simulation Technology website. [Online] Available: http://www.cst.de/

[4] H. Rahman et al., "Shielding effectiveness measurement techniques for various materials used for EMI shielding," in Proc. IEE Colloq. Screening 
of Connectors, Cables and Enclosures Digest No. 12, London, U.K., 1992, pp. $9 / 1-9 / 6$

[5] S. Kashyap, "Shielding effectiveness measurements with a dual TEM cell and a split TEM cell," in Proc. IEEE Int. Symp. Electromagn. Compat., San Diego, CA, 1986, pp. 262-264.

[6] ASTM D4935-99, Test Methodfor Measuring the Electromagnetic Shielding Effectiveness of Planar Materials, Amer. Soc. for Testing and Materials. West Conshohocken, PA, 1999.

[7] N. F. Colaneri and L. W. Shacklette, "EMI shielding measurements of conductive polymer blends," IEEE Trans. Instrum. Meas., vol. 41, no. 2, pp. 291-297, Apr. 1992.

[8] U. Lundgren, J. Ekman, and J. Delsing, "Characterization of conductive thermoplastic composite materials using multiple measurement methods," in Proc. EMC Europe 2002, Sorrento, Italy, Sep.

[9] MIL-STD 285, Method of Attenuation Measurement for Enclosures, Electromagnetic Shielding, for Electronic Test Purposes, U.S. Government Printing Office, Washington, DC, 1956.

[10] IEEE-STD 299, IEEE Standard Method for Measuring the Effectiveness of Electromagnetic Shielding Enclosures, IEEE, New York, 1997.

[11] P. F. Wilson and M. T. Ma, "Factors influencing material shielding effectiveness measurements," in Proc. IEEE EMC Soc. Symp., Boulder, CO, 1985, pp. 29-33.

[12] D. G. Bodnar, H. W. Denny, and B. M. Jenkins, "Shielding effectiveness measurements on conductive plastics," in Proc. IEEE EMC Soc. Symp., San Diego, CA, 1979, pp. 27-33.

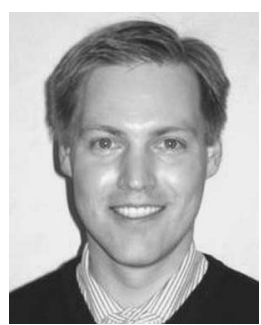

Urban Lundgren (S'98-A'02-M'04) received the M.Sc. degree in computer science and the Ph.D. degree in industrial electronics from Lulea University of Technology, Lulea, Sweden, in 1997 and 2004, respectively.

From 2004 to 2006, he was an electromagnetic compatibility (EMC) specialist at the BAE Systems Land Systems Hägglunds AB, Örnsköldsvik, Sweden. Currently, he is a consultant in EMC and industrial electronics design at the Omicron Ceti $\mathrm{AB}$, Umeå, Sweden. Since 1997, his research interests have been the measurement techniques for shielding effectiveness and electromagnetic parameters of materials in EMC issues.

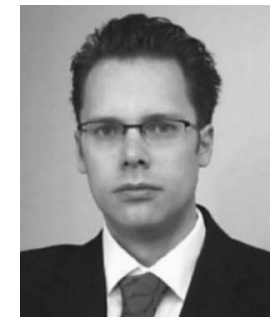

Jonas Ekman (A'03-M'02) was born in Boden, Sweden, in 1972. He received the Ph.D. degree in electrical engineering from Luleå University of Technology, Luleå, Sweden, in 2003.

During 2005 and 2006, he did his postdoctoral research in full-wave, time domain, PEEC modeling at the EMC Laboratory, University of L'Aquila, L'Aquila, Italy. Since 2003, he has been working as a Researcher at the EISLAB, Luleå University of Technology. His research interests are in computational electromagnetics, in particular, the use of the PEEC method for realistic electromagnetic modeling.

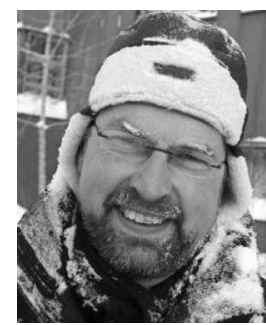

Jerker Delsing (S'85-M'88) received the M.Sc. degree in engineering physics and the docent degree in heat and power engineering from Lund Institute of Technology, Lund, Sweden, in 1982 and 1994, respectively, and the $\mathrm{Ph} . \mathrm{D}$. degree in electrical measurement from Lund University.

During 1985-1988, he worked part time at AlfaLaval-SattControl in the development of sensors and measurement technology. In 1995, he was a Full Professor of industrial electronics in Luleå University of Technology, Luleå, Sweden. Since 2004, he has been the Dean of Engineering Faculty at Luleå University of Technology. Currently, he the Scientific Head at the EISLAB, Luleå University of Technology. His current research interests are "Embedded Internet Systems," with applications to industry, medicine, and sports.

Dr. Delsing has been the President of the Instrument Tekniska Foreningen/Instrument Society of Sweden (ITF) since 1999. 